\title{
Toward Reliable and Energy Efficient Wireless Sensing for Space and Extreme Environments
}

\author{
Baek-Young Choi \\ Computer Science Electrical Engineering \\ University of Missouri - Kansas City \\ Kansas City, MO 64110, USA \\ Email: choiby@umkc.edu
}

\author{
Darren Boyd \\ NASA \\ Marshall Space and Flight Center \\ Huntsville AL 35812, USA \\ Email: darren.r.boyd@nasa.gov
}

\author{
DeLisa Wilkerson \\ NASA \\ Marshall Space and Flight Center \\ Huntsville AL 35812, USA \\ Email: delisa.l.wilkerson@nasa.gov
}

\begin{abstract}
Reliability is the critical challenge of wireless sensing in space systems operating in extreme environments. Energy efficiency is another concern for battery powered wireless sensors. Considering the physics of wireless communications, we propose an approach called Software-Defined Wireless Communications (SDC) that dynamically decide a reliable channel(s) avoiding unnecessary redundancy of channels, out of multiple distinct electromagnetic frequency bands such as radio and infrared frequencies. We validate the concept with Android and Raspberry Pi sensors and pseudo extreme experiments. SDC can be utilized in many areas beyond space applications.
\end{abstract}

\section{INTRODUCTION}

Sensing is integral for any space mission applications such as space vehicles, satellites and payloads, surface explorations, ground systems, and space habitats. Sensing data is the basis of monitoring and operation of the space missions. Sensing is also vital for exhaustive ground testings. Examples of various kinds of sensing needs are

- Physical sensing: temperature, humidity, pressure and radiation inside or around modules of a rocket, crew capsule, or habitat

- Chemical sensing: air quality and water quality of environment control and life support systems

- Biological sensing: mold, mildew or other airborne bacteria

- Crew health monitoring: vital signs and sleep behavior

- Ground testing: structure tolerance of test articles under stress from vibrations, physical strain, cryogenic and high temperatures
While there are some internal efforts being made to utilize wireless technologies for sensing, most sensing data transfer in space-related projects is currently done through wireline communication, as in Figure 1. It is because the space environment poses unique and extreme challenges such as radiations from solar events and cosmic rays, extremes temperatures, both hot and cold, depending its location relative to the Sun and due to absence of the insulating atmosphere of the Earth. In the midst of the harsh operational environment, reliability is a primary concern of NASA's missions, like the well-known quote, 'failure is not an option'[1]. Furthermore, a sensor's limited battery life is another challenge to stay operational for the duration of a mission.

On the other hand, for applications of the general public, there exists an enormous body of studies on wireless sensor networks including the issues of sensing coverage, routing, and architecture in the context of ad-hoc networks [2]. With the advancement of embedded systems and the prevalence of communication technologies, numerous Internet-of-Things (IoT) objects in everyday life are wirelessly connected to each other and through the Internet. The benefits of wireless sensors in space applications are tremendous and clear. The potential advantages of wireless sensing over wired counterparts include

- Reduced weight of spacecraft

- Easy and fast deployment

- Flexible sensor placement and data gathering from challenging area where wiring is difficult, hazardous or impossible

- Changes in location of sensors and data collectors

- Changes in the number of sensors and data collectors

- Reduced cost and better area usage

- Eliminated astronauts' time waste due to loose or lost cable

We propose a reliable and energy efficient wireless communication mechanism for sensors of space applications. We name the approach, Software-Defined Wireless Communication $(S D C)$. The idea stems from the rationale that redundancy is the fundamental approach at any level for reliability. While there have been many diversity mechanisms proposed for wireless communications, the focus has been limited to Radio Frequency (RF) spectrum at small frequency range levels. Considering the extreme conditions in space, we propose to 


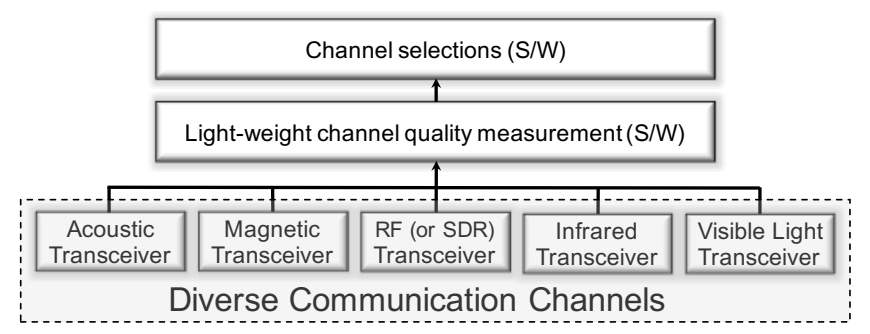

Fig. 2: The Concept of Software Defined Wireless Communication (SDC)

exploit multiple different electromagnetic (EM) spectrum that exhibit different physical characteristics such as RF, infrared (IR), visible lights (VL), etc. Through software mechanisms, the quality of each channel is monitored in a light-weight manner for reliability and energy efficiency, and the best channel is to be dynamically selected for data transmission depending on the environment channel conditions. To the best of our knowledge, this is the first work that dynamically uses diverse EM spectrum bands of different characteristics beyond RF.

\section{Software-Defined Wireless Communication (SDC) FOR RELIABLE AND ENERGY EFFICIENT WIRELESS COMMUNICATION}

There have been numerous technologies developed that adopt redundancy for improving the reliability of a wireless signal by using two or more communication channels with different RF bands. They exploit the diversities of time, frequency, space, multiuser, cooperation, or polarization. For instance, Software-Defined Radio (SDR) is a software approach to adaptively select and control appropriate communication radio frequency and corresponding physical layer modules such as detectors, filters, amplifiers, and modulators that have been traditionally implemented in hardware. Due to its software programmability, SDR can bridge multiple standards as well as legacy and future systems, and provides wireless communication capability that is interoperable with various communication devices operating on different RF band. Note that SDR devices such as how are relatively costly, depending on the ranges of RF spectrum observable and the options of configurable modules. Furthermore, the dynamic channel configuration in SDR is still limited only for a narrow scope of spectrum within RF range and does not cover infrared range of EM spectrum. However, existing technologies fundamentally address signal issues particular of wireless protocols of certain limited RF bands, and do not address interference issue of a large EM band.

The fundamental idea of the proposed Software Defined Wireless Communication (SDC) is to utilize multiple different EM bands redundantly that exhibit different physical characteristics such as one or more RF bands, Infrared (IR),

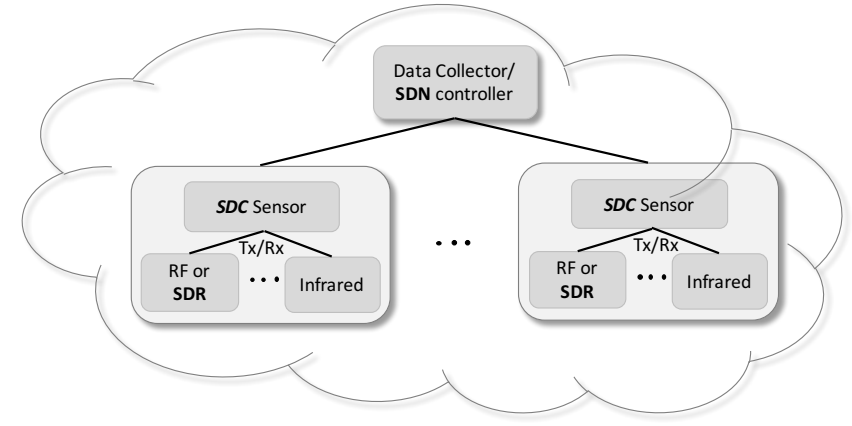

Fig. 3: Software-Defined Wireless Communication (SDC): Its relationship with SDN and SDR

and visible lights (VL). Acoustic or magnetic communication methods may potentially be used as well, depending on type and location of sensing (eg. liquid fuel sensing[3]). For energy efficiency, the most reliable channel among the available redundant channels is chosen at a given time. The quality of each channel is monitored in a light-weight manner in software (eg. periodic probing) where the quality can be defined based on the need of application, such as reliability, energy efficiency or their combinations. The quality of channels would vary dynamically subject to the channel environment conditions, and the best channel or top $k$ channels would be selected for data transmission. The rationale of choosing one or a few channels rather than the entire available ones is not to waste energy unnecessarily, and to cause unnecessary interference among sensors of the application. The concept is illustrated in Figure 2. To put in a relative perspective, SDC can utilize Software-Defined Radio (SDR) as one of its potential channel, and may be deployed in a Software-Defined Network (SDN) and/or in a network that uses Delay/Disruption Tolerance Network (DTN), as depicted in Figure 3.

As a proof-of-concept implementation, we have used an Android smartphone and a Raspberry Pi 3. Raspberry Pi 3 comes with Bluetooth and WiFi built-in, and we have it connected with IR sensor for infrared communication. Samsung smartphone Note 4 comes with equipped with RF (Bluetooth and WiFi) and Infrared emitter. We have developed infrared communication physical layer protocol similar to [4] and implemented an application that enables communications through either Bluetooth and/or IR. The poster will show the details and the performance evaluations in detail.

\section{REFERENCES}

[1] G. Kranz, Failure Is Not an Option: Mission Control From Mercury to Apollo 13 and Beyond. Simon and Schuster Publishing, 2000.

[2] A. Razi, F. Afghah, and A. Abedi, "Channel-Adaptive Packetization Policy for Minimal Latency and Maximal Energy Efficiency," IEEE Transactions on Wireless Communications, vol. 15, pp. 2407-2420, March 2016.

[3] G. Merrill, "Cryogenic Applications for Wireless Power and Data using Magnetics," in Proc. of IEEE International Conference on Wireless for Space and Extreme Environments (WISEE), 2015.

[4] K. Dhondge, K. Ayinala, B.-Y. Choi, and S. Song, "Optical Wireless Unlocking For Smart Door Locks Using Smartphones," in IEEE 12th International Conference on Mobile Ad-hoc and Sensor Networks (MSN), Hefei, China, December 2016. 